\title{
Spatially resolved GHz magnetization dynamics of a magnetite nano-particle chain inside a magnetotactic bacterium
}

\author{
Thomas Feggeler $\odot,{ }^{1, *}$ Ralf Meckenstock, ${ }^{1}$ Detlef Spoddig $\odot,{ }^{1}$ Benjamin W. Zingsem $\odot,{ }^{1,2}$ Hendrik Ohldag $\odot, 3,4, \dagger$

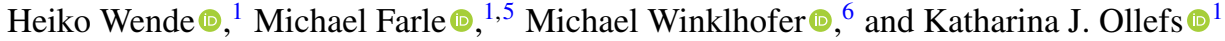 \\ ${ }^{1}$ Faculty of Physics and Center for Nanointegration Duisburg-Essen (CENIDE), University of Duisburg-Essen, 47048 Duisburg, Germany \\ ${ }^{2}$ Ernst Ruska Centre for Microscopy and Spectroscopy with Electrons and Peter Grünberg Institute, Forschungszentrum Jülich GmbH, \\ 52425 Jülich, Germany \\ ${ }^{3}$ SLAC National Accelerator Laboratory, Menlo Park, California 94025, USA \\ ${ }^{4}$ Department of Physics, University of California Santa Cruz, Santa Cruz, California 95064, USA \\ ${ }^{5}$ Kirensky Institute of Physics, Federal Research Center KSC SB RAS, Russia \\ ${ }^{6}$ School of Mathematics and Science, University of Oldenburg, 26129 Oldenburg, Germany
}

(Received 16 March 2021; accepted 14 June 2021; published 9 July 2021)

\begin{abstract}
Understanding magnonic properties of nonperiodic magnetic nanostructures requires real-space imaging of ferromagnetic resonance modes with spatial resolution well below the optical diffraction limit and sampling rates in the 5-100 GHz range. Here, we demonstrate element-specific scanning transmission $\mathrm{x}$-ray microscopydetected ferromagnetic resonance (STXM-FMR) applied to a chain of dipolarly coupled $\mathrm{Fe}_{3} \mathrm{O}_{4}$ nano-particles (40-50 nm particle size) inside a single cell of a magnetotactic bacterium Magnetospirillum magnetotacticum. The ferromagnetic resonance mode of the nano-particle chain driven at $6.748 \mathrm{GHz}$ and probed with $50 \mathrm{~nm}$ $\mathrm{x}$-ray focus size was found to have a uniform phase response but non-uniform amplitude response along the chain segments due to the superposition of dipolar coupled modes of chain segments and individual particles, in agreement with micromagnetic simulations.
\end{abstract}

DOI: 10.1103/PhysRevResearch.3.033036

\section{INTRODUCTION}

Magnonics deals with the controlled excitation and detection of spin waves in magnetic media [1-7]. Due to the low-energy nature of spin wave quanta (magnons), magnonic devices have been suggested to overcome key challenges of current charge-based computer information processing, such as power consumption and heat dissipation [8]. Recently, magnetic nano-particle chains were proposed as magnonic logic gates on the submicron scale, which would enable unprecedented transistor counts in a single central processing unit [9]. For visual control of the functionality of submicronsized magnon-based logic devices, it is essential to have a technique that can probe the magnetization dynamics on the $1-100 \mathrm{GHz}$ scale with few-nanometer resolution to determine the local phase and amplitude of excited magnons. Real-time observations using scanning electron microscopy

* Present address: Advanced Light Source, Lawrence Berkeley National Laboratory, Berkeley, California 94720, USA; tfeggeler@lbl.gov

${ }^{\dagger}$ Present address: Advanced Light Source, Lawrence Berkeley National Laboratory, Berkeley, California 94720, USA; and Department of Material Sciences and Engineering, Stanford University, Stanford, California 94305, USA.

Published by the American Physical Society under the terms of the Creative Commons Attribution 4.0 International license. Further distribution of this work must maintain attribution to the author(s) and the published article's title, journal citation, and DOI. with polarization analysis [10] have reached a temporal resolution (1 ns) sufficient to track relatively slow $\left(10^{2} \mathrm{MHz}\right)$ dynamic magnetization processes involving vortex cores in soft magnetic materials. Faster dynamical processes can be studied with pump-probe schemes repeated for a sequence of delay times with each repetition starting from the same initial state and accumulating many pump-probe pulse cycles in a stroboscopic procedure. Pump-probe schemes are used in time-resolved photoemission electron microscopy $[11,12]$ and ultrafast transmission electron microscopes $[13,14]$ capable of probing transient demagnetization dynamics of nanodiscs with sub-100 $\mathrm{nm}$ resolution and subpicosecond delay time resolution [15]. Here, we use scanning transmission x-ray microscopy-detected ferromagnetic resonance (STXM-FMR), where the magnetic specimen is driven by a continuous wave excitation, while temporal sampling is performed stroboscopically at a higher harmonic $[16,17]$. The technique offers element specificity, $\leqslant 50 \mathrm{~nm}$ spatial resolution, and sampling rates of tens of $\mathrm{GHz}$, allowing for spatial detection of resonant responses at up to $10 \mathrm{GHz}$ [16]. By exploiting these features of STXM-FMR, we were able to detect a spatially nonuniform ferromagnetic resonance mode across a single chain of magnetite $\left(\mathrm{Fe}_{3} \mathrm{O}_{4}\right)$ nano-particles in a magnetotactic bacterium. Thus, we demonstrate the feasibility to detect ferromagnetic resonance in a nanomagnetic system with $\leqslant 50 \mathrm{~nm}$ spatial resolution.

\section{EXPERIMENTAL DETAILS}

With the STXM-FMR setup at the Stanford Synchrotron Radiation Lightsource (SSRL), FMR excitations in the linear 

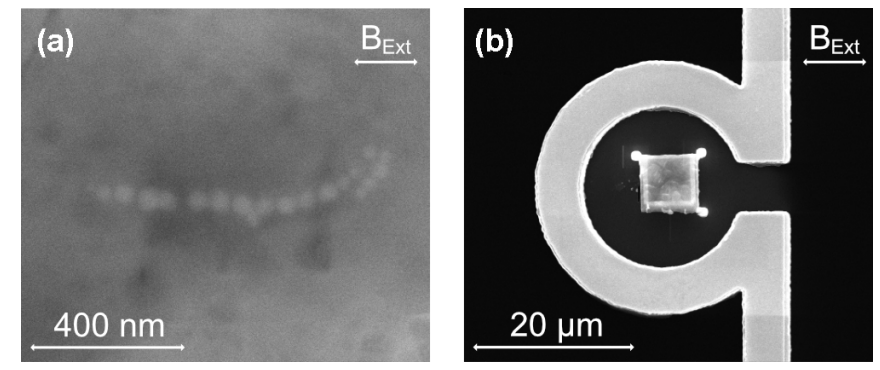

FIG. 1. (a) Electron micrograph of the $\mathrm{Fe}_{3} \mathrm{O}_{4}$ nano-particle chain selected for scanning transmission $\mathrm{X}$-ray microscopy-detected ferromagnetic resonance (STXM-FMR). The structure was deposited on a transmission electron microscopy (TEM) grid and cut out with a focused ion beam (FIB). (b) Cutout area including the nano-particle chain positioned in the center of the microresonator (light gray "omega"-shaped Au conduit). The direction of the magnetic field $B_{\text {Ext }}$ is indicated.

regime are measured element-specific by employing the $\mathrm{x}$-ray magnetic circular dichroism (XMCD) effect $[18,19]$ in the transverse XMCD geometry [20]. The XMCD effect, defined as difference in $\mathrm{X}$-ray absorption of left- and right-hand circularly polarized $\mathrm{x}$ rays, is a measure of the magnetization $M$ of the probed material projected onto the $\mathbf{k}$ vector of the incident $\mathrm{x}$ rays. The resonant magnetic excitation is realized by applying linearly polarized continuous microwaves $b_{\mathrm{mw}}$ to the sample, polarized parallel to the propagation direction of the $\mathrm{x}$ rays, while the static magnetic field $B_{\mathrm{Ext}}$ is orientated perpendicular to the $\mathbf{k}$ vector of the $\mathrm{x}$ rays. For the homogeneous excitation with high conventional detection sensitivity, a microresonator setup is used [21].

The sample contains a chain of $19 \mathrm{Fe}_{3} \mathrm{O}_{4}$ [22-25] nanoparticles with particle sizes of $40-50 \mathrm{~nm}$ [25] arranged in a segment of 15 particles and a smaller segment of four particles offset from this segment by a distance of $\sim 25 \mathrm{~nm}$ [Fig. 1(a)]. The nano-particle chain grew naturally by biomineralization inside a bacterial cell of Magnetospirillum magnetotacticum [25-27]. The bacteria were obtained from Leibniz Institute DSMZ - German Collection of Microorganisms and Cell Cultures [28] as actively growing culture and positioned on a transmission electron microscopy (TEM) grid [Fig. 1(a)]. The grid with the nano-particles was cut out by focused ion beam (FIB) milling and placed at the center of the microresonator loop [Fig. 1(b)]. The external magnetic field $B_{\mathrm{Ext}}=0.167 \pm$ $0.002 \mathrm{~T}$ (Fig. 1), corresponding to the resonance field predicted by our micromagnetic simulations, was applied in the sample plane along the direction of the chain axis, as indicated in Fig. 1. The sample was probed under dry conditions at room temperature at the $\mathrm{Fe} L_{3}$-edge (708 eV [29]) with a focus of $\leqslant 50 \mathrm{~nm}$, a step size of $25 \mathrm{~nm}$, and a dwell time of $5 \mathrm{~s}$ per pixel with constant polarization of the $\mathrm{x}$ rays. The microwave excitation at $6.748 \mathrm{GHz}$ with a power of $25 \mathrm{dBm}$ was chosen as the $\left(14+\frac{1}{6}\right)$ th harmonics of the synchrotron frequency.

In the STXM-FMR measurements, two sets of six consecutive images each are recorded pixel by pixel, which correspond to six images recorded with microwaves on and six images with microwaves off, respectively. Between two consecutive images, there is a time difference of $24.7 \mathrm{ps}$ and a phase difference of $\Delta \varphi=60^{\circ}$, yielding to the monitoring
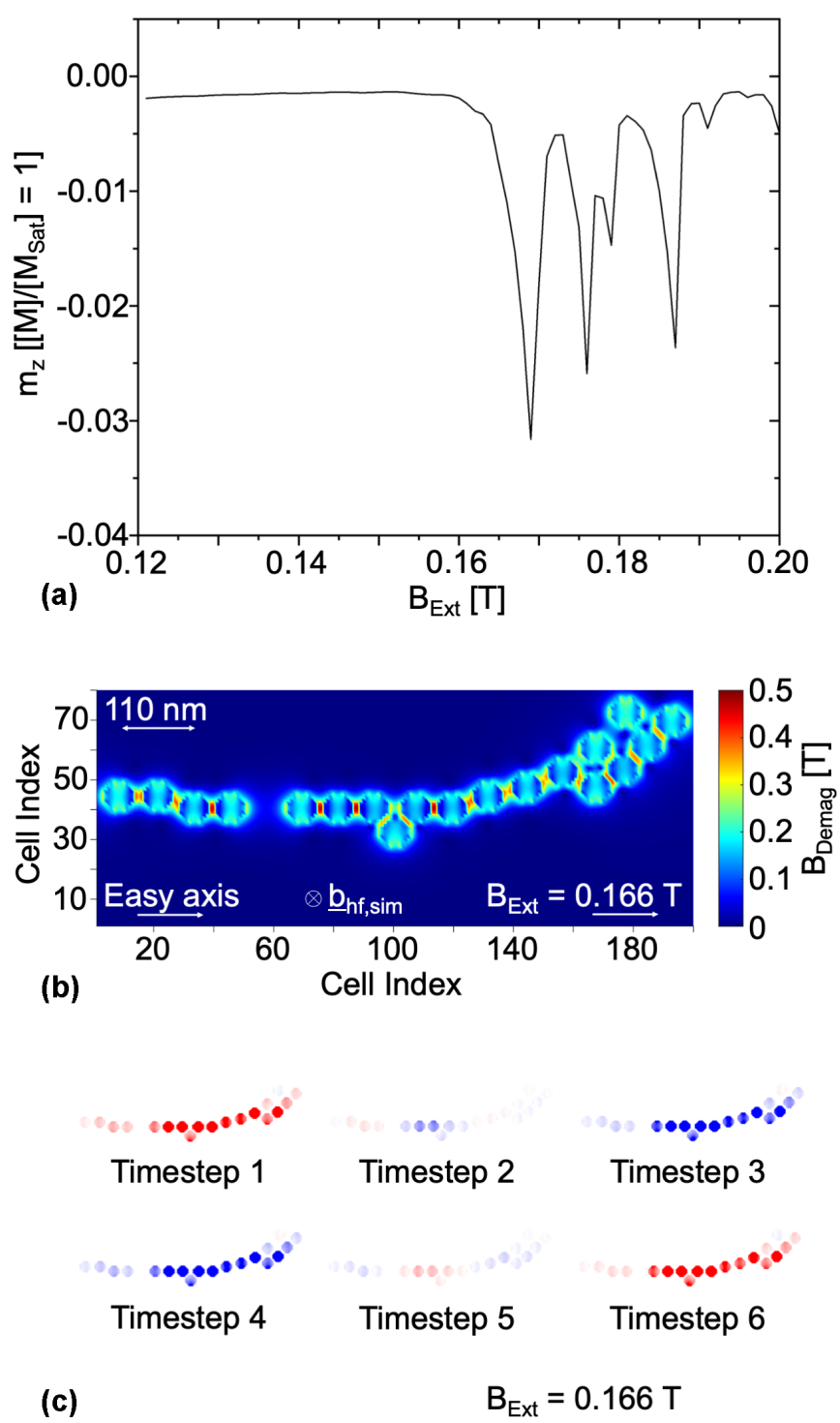

FIG. 2. (a) Simulated ferromagnetic resonance (FMR) absorption spectrum in terms of the normalized $m_{z}$ component of the magnetization over the field range $B_{\mathrm{Ext}}=0.12-0.2 \mathrm{~T}$. (b) Spatial distribution of the simulated inner demagnetization and outer stray field at an applied static magnetic bias field of 0.166 T. (c) Six time steps ( $\Delta t \approx 24.7 \mathrm{ps}$ ) of the dynamic micromagnetic simulation, displaying a spatially inhomogeneous oscillation response. The normalized $m_{z}$ out-of-plane component of the magnetization is color coded $(-z$ : blue, $+z$ : red).

of six time steps of the microwave cycle [16]. To extract the microwave-induced $\mathrm{x}$-ray transmission, the natural logarithm of the microwave-off data divided by the microwave-on data was taken. The data were normalized to the average intensity with consecutive minimum-maximum normalization. The phase and amplitude response at each pixel was obtained by fitting a sine function to the time-dependent data [30]. The result is coded in the hue-saturation-brightness (HSB) format to represent the phase relative to the time $t=0 \mathrm{ps}$ as hue [31], the amplitude as brightness, and the fit accuracy as saturation [30]. 


\section{Experiment}
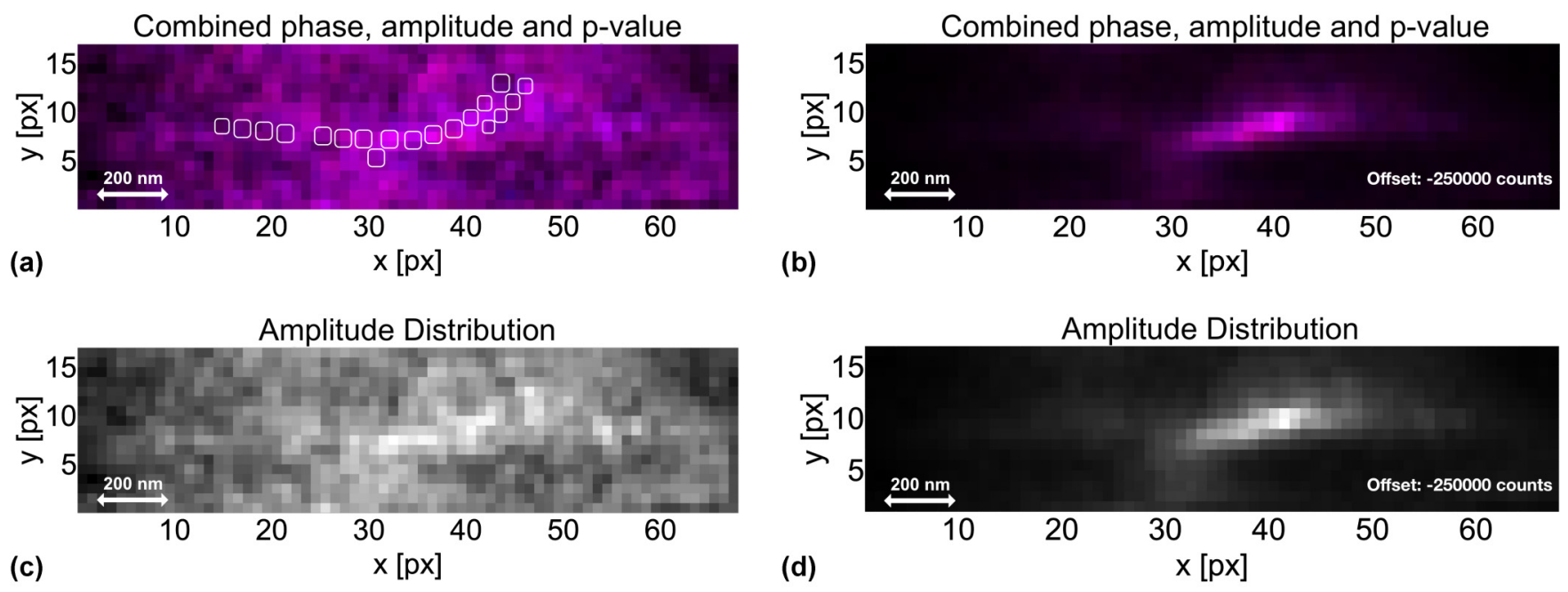

\section{Micromagnetic Simulation}

Phase [deg.]
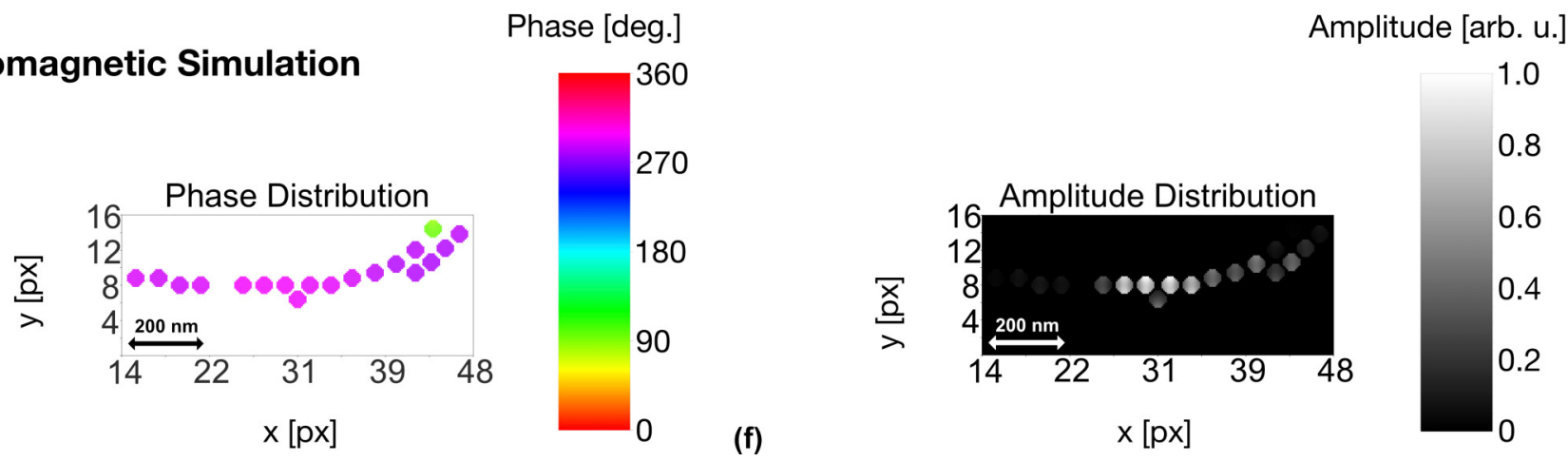

FIG. 3. (a) Result of the pixel-by-pixel sinusoidal fit analysis for the scanning transmission x-ray microscopy-detected ferromagnetic resonance (STXM-FMR) data at $B_{\mathrm{Ext}}=167 \mathrm{mT}$ at $f=6.748 \mathrm{GHz}$. The phase relative to $t=0 \mathrm{ps}$, the amplitude, and the fit accuracy are coded as hue, brightness, and saturation. The estimated particle positions are indicated by rounded squares. (b) Subfigure (a) after subtraction of an offset of 250000 counts. (c) and (d) Normalized amplitude distribution extracted from (a) and (b) respectively. (e) and (f) Phase and amplitude distribution of the micromagnetic simulation at $B_{\text {Ext }}=166 \mathrm{mT}$ at $f=6.748 \mathrm{GHz}$.

From the scanning electron micrographs [Fig. 1(a)], we designed a three-dimensional (3D) computer model of the nano-particle chain (single particle size $50 \mathrm{~nm}$ ) using the micromagnetic simulation package MUMAX3 $[32,33]$. Considering the not perfectly stoichiometric $\mathrm{Fe}_{3} \mathrm{O}_{4}$ with the known decrease of the Verwey transition temperature [34], the saturation magnetization $M_{\text {Sat }}$, and the magnetocrystalline anisotropy constant $K_{1}$ [35], the dynamic simulation was calculated according to Ref. [35] with $M_{\text {Sat }}=465 \mathrm{kA} / \mathrm{m}$, and a first-order cubic anisotropy constant $K_{1}=-1.0 \times 10^{4} \mathrm{~J} / \mathrm{m}_{3}$. The exchange stiffness was set to $A=1.32 \times 10^{-11} \mathrm{~J} / \mathrm{m}[36]$, and the simulation grid was defined as $200 \times 80 \times 12$ cells with a cell size of $(5 \mathrm{~nm})^{3}$.

\section{RESULTS AND DISCUSSION}

In the FMR absorption spectrum simulated at $6.748 \mathrm{GHz}$ [Fig. 2(a)], we observe three resonances at $0.169 \mathrm{~T}, 0.176 \mathrm{~T}$, and $0.187 \mathrm{~T}$, which is surprising, given that a linear chain usually behaves as an uniaxial magnet with one resonance mode per field orientation [37]. Evidently, variations in the orientation and strength of the dipolar coupling between the particles in a chain produce complex magnon dispersion rela- tions with multiple resonance lines and even magnonic band gaps, where resonances involving the same group of particles have incommensurate spatial mode profiles [9]. The variations in dipolar coupling are most pronounced in chain geometries with irregular particle spacings [9]. Indeed, for the chain at hand, the micromagnetic simulation confirms pronounced variations in dipolar coupling strength [Fig. 2(b)] along the chain axis, ranging from $0.450 \mathrm{~T}$ between adjacent particles (orange/red color) down to $0.050 \mathrm{~T}$ in the gap between the two chain segments. This essentially dynamically decouples the small four-particle segment on the left from the main chain segment. The decoupling becomes apparent in the snapshots of the dynamic micromagnetic simulation [Fig. 2(c)], where the oscillation amplitude is large in the main segment and small in the four-particle segment. Likewise, at the far right end of this chain, the oscillation amplitudes are comparatively small [Fig. 2(c)], which is due to the disturbance of the simple linear chain structure by two pairs of particles in side-by-side position perpendicular to the chain axis. In both side-by-side pairs, it is the lower particle that is more strongly coupled to the curved chain axis [compare with Fig. 2(b)], so that it can be excited more easily in the main resonance mode of the chain. Thus, on the basis of our micromagnetic simulations, 
we expect a nonhomogeneous microwave response along the particle chain.

Indeed, the experimentally determined resonance amplitudes [Figs. 3(a) and 3(c)] are strongest (high brightness purple, white) in the main segment of the chain, but almost vanish in the left four-particle segment extending from $x=14$ to $22 \mathrm{px}$ at $y=10 \mathrm{px}$. Although individual particles cannot be resolved due to the limited focus $(50 \mathrm{~nm})$ at the $\mathrm{Fe} L_{3}$, the upcurving bright white intensity pattern $(x=25-48$ px and $y$ $=6-12 \mathrm{px}$ ) tracks the curvature of the main segment of the chain. We note that, in Figs. 3(a) and 3(c), we find a nonzero phase and amplitude not only at the location of the chain but almost everywhere in the field of view. The reason for this observation is that there is small crosstalk between the applied microwave and the (shielded) avalanche photodiode. Since the setup described in Ref. [16] represents a high-quality factor lock-in amplifier tuned to the exciting microwave frequency, even the smallest fraction of microwave power at the diode will be detected. In consequence, also without a sample, a nonzero signal is observed. To remove the visibility of this background, we discriminate the detected channel with a value of 250000 counts (see the Supplemental Material [38]), revealing a clearly distinguishable resonant response of the particle chain with the highest amplitude along the upcurving chain segment. The phase map [Figs. 3(a) and 3(b)] reveals a largely uniform resonant response of the particle chain $\left(270^{\circ}\right.$ to $300^{\circ}$ ) and is consistent with the time snapshots of the micromagnetic simulation [Fig. 2(c)]. For comparison between experiment and theory, we evaluated the snapshot sequences from the micromagnetic simulations in the same way as the experimental image sequences to obtain phase and amplitude maps [Figs. 3(e) and 3(f)]. All simulation results are pictured at $B_{\text {Ext }}=166 \mathrm{mT}$, at which the best match to the experimental data is observed. Deviations between calculated and observed maps can be ascribed to the fact that not all small features of the 3D particles are reflected in the computer model, resulting in equally sized particles in the micromagnetic model, while the particle sizes and spacing in the real chain have some variability, particularly at the right end of the chain. This leads to variations in dipolar coupling strength [compare Fig. 2(b)] and, in consequence, in the observed resonance modes.
The calculated phase response tallies with the observed one, except for the particle at the far upper right, which oscillates antiphasic to the rest of the chain [Fig. 3(e)], albeit with very low amplitude [Fig. 3(f)]. As can be seen in the amplitude map, the response of the left four-particle segment indeed is weak, which explains the lack of signal in this region in the measured amplitude map. As inferred from the stray field map above [Fig. 2(b)], the particles that are vertically offset from the central chain axis and more weakly coupled to the chain are weakly excited and thus have low oscillation amplitudes [Fig. 3(f)].

In summary, we observe a uniform phase but nonuniform amplitude response of the nano-particle chain. With the STXM-FMR technique, we thus demonstrate a measurement of a ferromagnetic resonance mode of a nano-particle chain in a spatially resolved manner $(\leqslant 50 \mathrm{~nm})$. This technique allows us to element-specifically examine the functionality of both periodic and nonperiodic magnetic nanostructures of future magnonic and spintronic devices, like for magnon spin transistors [39] and biomagnonics [9,40], complementing spatially resolved, nonelement-specific, experimental techniques and micromagnetic simulations. A STXM-FMR setup taking advantage of the increased spatial resolution of $10 \mathrm{~nm}$ [41] at the Advanced Light Source (ALS) will open the pathway to image $\mathrm{x}$-band $\mathrm{GHz}$ magnetization dynamics of nanostructures on the $\leqslant 20 \mathrm{~nm}$ scale.

\section{ACKNOWLEDGMENTS}

This work is funded by the Deutsche Forschungsgemeinschaft (DFG, German Research Foundation) - Project No. OL513/1-1, 321560838, and in part by the Deutsche Forschungsgemeinschaft (DFG, German Research Foundation) - Project-ID 405553726 TRR 270. M.F. acknowledges support by the government of the Russian Federation (agreement No. 075-15-2019-1886). We gratefully acknowledge A. Ney for fruitful discussions. The use of the Stanford Synchrotron Radiation Lightsource, SLAC National Accelerator Laboratory, is supported by the US Department of Energy, Office of Science, Office of Basic Energy Sciences under Contract No. DE-AC02-76SF00515.
[1] A. V. Chumak, A. A. Serga, and B. Hillebrands, Magnonic crystals for data processing, J. Phys. D: Appl. Phys. 50, 244001 (2017).

[2] A. V. Chumak, V. I. Vasyuchka, and B. Hillebrands, Magnon spintronics, Nat. Phys. 11, 453 (2015).

[3] S. Urazhdin, V. E. Demidov, H. Ulrichs, T. Kendziorczyk, T. Kuhn, H. Leuthold, G. Wilde, and S. O. Demokritov, Nanomagnonic devices based on the spin-transfer torque, Nat. Nanotech. 9, 509 (2014).

[4] K. Vogt, F. Y. Fradin, J. E. Pearson, S. D. Bader, B. Hillebrands, A. Hoffmann, and H. Schultheiss, Realization of a spin-wave multiplexer, Nat. Commun. 5, 3727 (2014).

[5] N. Sato, K. Sekiguchi, and Y. Nozaki, Electrical demonstration of spin-wave logic operation, Appl. Phys. Exp. 6, 063001 (2013).
[6] V. V. Kruglyak, S. O. Demokritov, and D. Grundler, Magnonics, J. Phys. D: Appl. Phys. 43, 264001 (2010).

[7] A. Khitun, M. Bao, and K. L. Wang, Magnonic logic circuits, J. Phys. D: Appl. Phys. 43, 264005 (2010).

[8] P. J. Denning and T. G. Lewis, Exponential laws of computing growth, Commun. ACM 60, 54 (2016).

[9] B. W. Zingsem, T. Feggeler, A. Terwey, S. Ghaisari, D. Spoddig, D. Faivre, R. Meckenstock, M. Farle, and M. Winklhofer, Biologically encoded magnonics, Nat. Commun. 10, 4345 (2019).

[10] R. Frömter, F. Kloodt, S. Rößler, A. Frauen, P. Staeck, D. R. Cavicchia, L. Bocklage, V. Röbisch, E. Quandt, and H. P. Oepen, Time-resolved scanning electron microscopy with polarization analysis, Appl. Phys. Lett. 108, 142401 (2016). 
[11] A. Krasyuk, A. Oelsner, S. A. Nepijko, A. Kuksov, C. M. Schneider, and G. Schönhense, Time-resolved photoemission electron microscopy of magnetic field, and magnetization changes, Appl. Phys. A-Mater. 76, 863 (2003).

[12] J. Vogel, W. Kuch, M. Bonfim, J. Camarero, Y. Pennec, F. Offi, K. Fukumoto, J. Kirschner, A. Fontaine, and S. Pizzini, Time-resolved magnetic domain imaging by x-ray photoemission electron microscopy, Appl. Phys. Lett. 82, 2299 (2003).

[13] H. S. Park, J. Spencer Baskin, and A. H. Zewail, 4D Lorentz electron microscopy imaging: magnetic domain wall nucleation, reversal, and wave velocity, Nano Lett. 10, 3796 (2010).

[14] K. B. Schliep, P. Quarterman, J.-P. Wang, and D. J. Flannigan, Picosecond Fresnel transmission electron microscopy, Appl. Phys. Lett. 110, 222404 (2017).

[15] N. Rubiano da Silva, M. Möller, A. Feist, H. Ulrichs, C. Ropers, and S. Schäfer, Nanoscale Mapping of Ultrafast Magnetization Dynamics with Femtosecond Lorentz Microscopy, Phys. Rev. X 8, 031052 (2018).

[16] S. Bonetti, R. Kukreja, Z. Chen, D. Spoddig, K. Ollefs, Ch. Schöppner, R. Meckenstock, A. Ney, J. Pinto, R. Houanche, J. Frisch, J. Stöhr, H. A. Dürr, H. Ohldag, Microwave soft $\mathrm{x}$-ray microscopy for nanoscale magnetization dynamics in the 5-10 GHz frequency range, Rev. Sci. Instrum. 86, 093703 (2015).

[17] T. Schaffers, R. Meckenstock, D. Spoddig, T. Feggeler, K. Ollefs, Ch. Schöppner, S. Bonetti, H. Ohldag, M. Farle, and A. Ney, The combination of micro-resonators with spatially resolved ferromagnetic resonance, Rev. Sci. Instrum. 88, 093703 (2017).

[18] H. A. Dürr, T. Eimüller, H.-J. Elmers, S. Eisebitt, M. Farle, W. Kuch, F. Matthes, M. Mertins, H.-C. Mertins, P. M. Oppeneer, L. Plucinski, C. M. Schneider, H. Wende, W. Wurth, and H. Zabel, A closer look into magnetism: opportunities with synchrotron radiation, IEEE Trans. Magn. 45, 15 (2009).

[19] G. van der Laan and A. I. Figueroa, X-ray magnetic circular dichroism - a versatile tool to study magnetism, Coordin. Chem. Rev. 277, 95 (2014).

[20] J. Goulon, A. Rogalev, F. Wilhelm, N. Jaouen, C. GoulonGinet, and C. Brouder, X-ray detected ferromagnetic resonance in thin films, Eur. Phys. J. B 53, 169 (2006).

[21] R. Narkowicz, D. Suter, and R. Stonies, Planar microresonators for EPR experiments, J. Magn. Reson. 175, 275 (2005).

[22] X. Zhu, A. P. Hitchcock, D. A. Bazylinski, P. Denes, J. Joseph, U. Lins, S. Marchesini, H. W. Shiu, T. Tyliszczak, and D. A. Shapiro, Measuring spectroscopy, and magnetism of extracted, and intracellular magnetosomes using soft x-ray ptychography, Proc. Natl. Acad. Sci. USA 113, E8219 (2016).

[23] M. Posfai, C. T. Lefèvre, D. Trubitsyn, D. A. Bazylinski, and R. B. Frankel, Phylogenetic significance of composition, and crystal morphology of magnetosome minerals, Front. Microbiol. 4, 1 (2013).

[24] R. B. Frankel, R. Blakemore, and R. S. Wolfe, Magnetite in freshwater magnetotactic bacteria, Science 203, 1355 (1979).

[25] D. Maratea and R. Blakemore, Aquaspirillum magnetotacticum sp. nov., a magnetic spirillum, Int. J. Syst. Bacteriol. 31, 452 (1981)
[26] D. Faivre and D. Schüler, Magnetotactic bacteria and magnetosomes, Chem. Rev. 108, 4875 (2008).

[27] K. H. Schleifer, D. Schüler, S. Spring, M. Weizenegger, R. Amann, W. Ludwig, and M. Köhler, The genus Magnetospirillum gen. nov., description of Magnetospirillum gryphiswaldense sp. nov., and transfer of Aquaspirillum magnetotacticum to Magnetospirillum magnetotacticum comb. nov., Syst. Appl. Microbiol. 14, 379 (1991).

[28] Leibniz Institute DSMZ. German Collection of Microorganisms and Cell Cultures $\mathrm{GmbH}$, Magnetospirillum magnetotacticum DSM 3856, https://www.dsmz.de/collection/catalogue/details/ culture/DSM-3856, last time accessed 06/28/2021,

[29] A. C. Thompson, J. Kirz, D. T. Attwood, E. M. Gullikson, M. R. Howells, J. B. Kortright, Y. Liu, A. L. Robinson, J. H. Underwood, K.-J. Kim, I. Lindau, P. Pianetta, H. Winick, G. P. Williams, and J. H. Scofield, X-Ray Data Booklet, 3rd ed. (Lawrence Berkeley National Laboratory University of California, Berkeley, 2009).

[30] B. Zingsem, T. Feggeler, R. Meckenstock, T. Schaffers, S. Pile, H. Ohldag, M. Farle, H. Wende, A. Ney, and K. Ollefs, Evaluation protocol for revealing magnonic contrast in STXM-FMR measurements, arXiv:1901.10595 [cond-mat.str-el], 2019.

[31] M. D. Fairchild, Color Appearance Models, 3rd ed. (John Wiley $\&$ Sons, Chichester, 2013).

[32] A. Vansteenkiste, J. Leliaert, M. Dvornik, M. Helsen, F. GarciaSanchez, and B. Van Waeyenberge, The design and verification of MuMax3, AIP Adv. 4, 107133 (2014).

[33] L. Exl, S. Bance, F. Reichel, T. Schrefl, H. P. Stimming, and N. J. Mauser, LaBonte's method revisited: an effective steepest descent method for micromagnetic energy minimization, J. Appl. Phys. 115, 17D118 (2014).

[34] R. Aragón, D. J. Buttrey, J. P. Shepherd, and J. M. Honig, Influence of nonstoichiometry on the Verwey transition, Phys. Rev. B 31, 430 (1985).

[35] Z. Kąkol and J. M. Honig, Influence of deviations from ideal stoichiometry on the anisotropy parameters of magnetite $\mathrm{Fe}_{3(1-\delta)} \mathrm{O}_{4}$, Phys. Rev. B 40, 9090 (1989).

[36] H.-C. Wu, S. K. Arora, O. N. Mryasov, and I. V. Shvets, Antiferromagnetic interlayer exchange coupling between $\mathrm{Fe}_{3} \mathrm{O}_{4}$ layers across a nonmagnetic $\mathrm{MgO}$ dielectric layer, Appl. Phys. Lett. 92, 182502 (2008).

[37] M. Charilaou, M. Winklhofer, and A. U. Gehring, Simulation of ferromagnetic resonance spectra of linear chains of magnetite nanocrystals, J. Appl. Phys. 109, 093903 (2011).

[38] See Supplemental Material at http://link.aps.org/supplemental/ 10.1103/PhysRevResearch.3.033036 for experimental information on the time-resolved setup and considerations on the oscillating background.

[39] A. V. Chumak, A. a. Serga, and B. Hillebrands, Magnon transistor for all-magnon data processing, Nat. Commun. 5, 4700 (2014).

[40] A. Barman et al., The 2021 magnonics roadmap, J. Phys.: Condens. Matter to be published (2021).

[41] W. Chao, P. Fischer, T. Tyliszczak, S. Rekawa, E. Anderson, and P. Naulleau, Real space soft X-ray imaging at $10 \mathrm{~nm}$ spatial resolution, Opt. Express 20, 9777 (2012). 